\title{
Longitudinal Follow-Up and Outcomes for Chinese Patients with Stage 1-4 Chronic Kidney Disease
}

\author{
Jinwei Wang ${ }^{a, b}, c, d, e$ Jicheng Lva,b, c, d, e Kevin He ${ }^{f}$ Fang Wang ${ }^{a, b}, c, d, e$ \\ Bixia Gao a, b, c, d, e Ming-Hui Zhao a, b, c, d, e, g Luxia Zhang a, b, c, d, e, h, i \\ the Chinese Cohort Study of Chronic Kidney Disease (C-STRIDE) collaborators
}

\begin{abstract}
${ }^{a}$ Renal Division, Department of Medicine, Peking University First Hospital, Beijing, China; ${ }^{\mathrm{b}}$ Institute of Nephrology, Peking University, Beijing, China; ${ }^{\complement}$ Key Laboratory of Renal Disease, Ministry of Health of China, Beijing, China; dKey Laboratory of Chronic Kidney Disease Prevention and Treatment (Peking University), Ministry of Education of

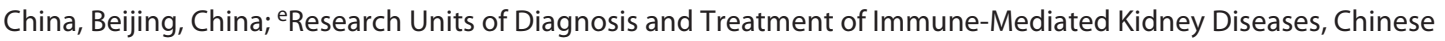
Academy of Medical Sciences, Beijing, China; fDepartment of Biostatistics, School of Public Health, University of Michigan, Ann Arbor, MI, USA; ${ }^{9}$ Peking-Tsinghua Center for Life Sciences, Beijing, China; ${ }^{h}$ Center for Data Science in Health and Medicine, Peking University, Beijing, China; 'National Institute of Health Data Science at Peking University, Beijing, China
\end{abstract}

\section{Keywords}

All-cause mortality · Cardiovascular disease $\cdot$ Chronic kidney disease $\cdot$ Cohort $\cdot$ End-stage kidney disease $\cdot$ Incidence rate

\begin{abstract}
Introduction: Understanding heterogeneity in the prognosis of chronic kidney disease (CKD) has implications in management of patients. We aimed to evaluate the comparative risk of end-stage kidney disease (ESKD), cardiovascular (CV) events, and death among patients with CKD in China. Methods: In total, 3,700 patients with CKD stage 1-4 were recruited from 39 clinical centers in China between 2011 and 2016. New occurrence of ESKD, CV events, and all-cause mortality was recorded until the end of 2017. The crude incidence rate was calculated for each outcome. Ratios of incidence between different outcomes were generated with 95\% confidence interval $(\mathrm{Cl})$ estimated by 1,000 times of bootstrapping. Multivariable adjusted Cox regression models accounting for competing risk between the outcomes were used to evaluate the association of risk factors with the outcomes.
\end{abstract}

Results: The population mean age was $50 \pm 14$ years, with $58.2 \%$ male and $60.3 \%$ of glomerulonephritis. After a median follow-up of 4.65 years (interquartile range [IQR]: 3.71-5.60 years) for ESKD, 4.76 years (IQR: 3.97-5.76 years) for CV events, and 4.84 years (IQR: 3.97-5.76 years) for death, the incidence rates of the 3 outcomes were $3.1,1.5$, and $0.92 / 100$ patient-years, respectively. The ratio for the incidence of ESKD and CV events was 2.15 (95\% Cl: 1.87, 2.53) and that for incidence of ESKD and death was 3.41 (95\% Cl: 2.88, 4.08). Significant differences regarding the ratios were detected through levels of age, history of CV disease, the estimated glomerular filtration rate (eGFR), urine albumin-to-creatinine ratio ( $\mathrm{UACR}$ ), and etiology of CKD. In the Cox regression model adjusting for traditional CV and kidney-specific risk factors, older age was associated with a higher risk of CV events and death but a lower risk of ESKD (hazard ratios [HRs] $=1.45$ [95\% Cl: 1.29, 1.64], 1.48 [95\% Cl: 1.29, 1.70], and 0.78

The Chinese Cohort Study of Chronic Kidney Disease (C-STRIDE) collaborators are listed in the online supplementary file (see www.karger.com/doi/10.1159/000519190). karger@karger.com www.karger.com/kdd

Karger $\frac{1}{\%}$

GOPEN ACCESS
(C) 2021 The Author(s)

Published by S. Karger AG, Basel

This is an Open Access article licensed under the Creative Commons Attribution-NonCommercial-4.0 International License (CC BY-NC) (http://www.karger.com/Services/OpenAccessLicense), applicable to the online version of the article only. Usage and distribution for commercial purposes requires written permission.
Correspondence to:

Luxia Zhang, zhanglx@bjmu.edu.cn 
[95\% Cl: 0.73, 0.84] per 10 year increase, respectively). By comparison, reduced eGFR was associated with a higher risk of ESKD and death, rather than CV events (HRs = $3.62[95 \%$ Cl: 2.96, 4.43], 1.30 [95\% Cl: 1.02, 1.66], and 1.22 [95\% Cl: 0.99 , 1.49] per $30.26 \mathrm{~mL} / \mathrm{min} / 1.73 \mathrm{~m}^{2}$ increase, respectively). Similar patterns were seen for increased uACR (HRs $=1.42[95 \%$ $\mathrm{Cl}: 1.30,1.55], 1.17$ [95\% Cl: 1.05, 1.30], and 1.07 [95\% Cl: 0.99, 1.17] per 1 natural log-transformed value increase, respectively). Conclusion: ESKD was more likely to occur than CV events and death in the population with CKD stage 1-4 in China. Traditional risk factors contributed differently to the comparative risk of the outcomes. @ 2021 The Author(s).

Published by S. Karger AG, Basel

\section{Introduction}

Chronic kidney disease (CKD) is a global public health problem, leading to an increased morbidity and mortality [1]. Understanding the comparative risk for different outcomes of CKD has implications for the effective management of patients and health policy making. Several large managed care program-based studies have focused on the topic and provided the evidence that patients with predialysis CKD are far more likely to die or have cardiovascular $(\mathrm{CV})$ events than to progress to end-stage kidney disease (ESKD) [2-4]. However, hospital-based cohort studies recruiting patients of CKD reported a highly heterogeneous pattern for the different outcomes of CKD. Some of the cohorts, such as the Australia-based CKD in Queensland study and the National Renal Healthcare Program of Uruguay, still reported a higher risk of ESKD than all-cause mortality and CV events, but the gap of incidence between the outcomes was not as large as that observed in the managed care program-based studies [5]. In contrast, other studies, like the African American Study of Kidney Disease and Hypertension (AASK) study and the CKD-Japan cohort demonstrated that the incidence rate of ESKD exceeded that of all-cause mortality or composite CV events [6,7]. Diversities in ethnicity, metabolic risk factors, and access to health care may contribute to the variation of comparative risk of different outcomes of CKD.

The spectrum for etiology of CKD in China differs from that in Western countries. Although the increasing prevalence of diabetes from 1980s has led to more patients affected by diabetic kidney disease, glomerulonephritis may still account for a considerate proportion of patients with CKD in China $[8,9]$. As there are currently no comprehensive registries of CKD in China, the pattern

Comparative Risk for Outcomes of

Chronic Kidney Disease in China for the prognosis and outcomes of CKD in the country is not well understood. In addition, the impacts of risk factors on the development of outcomes may be heterogeneous, clarification of which may shed light on mechanisms of the progression of CKD and help improve management of patients.

In this study, we examined the prognosis of CKD in China, using the Chinese cohort study of CKD (CSTRIDE), where ESKD, CV events, and death are well recorded. In addition, we evaluated the association of traditional $\mathrm{CV}$ risk factors and kidney-specific measures with the outcomes.

\section{Materials and Methods}

\section{Participants}

C-STRIDE is an ongoing cohort study initiated in November 2011, which includes patients with stage $1-4$ CKD and various etiologies in 39 tertiary academic hospitals around China. The detailed study design and methods have been published previously $[10,11]$. There are 2 criteria to be eligible for enrollment. First, participants should be adults aged 18-74 years. Second, the estimated glomerular filtration rate (eGFR) should be in the specific range according to different CKD etiologies. For patients with glomerulonephritis, eGFR should be $\geq 15 \mathrm{~mL} / \mathrm{min} / 1.73 \mathrm{~m}^{2}$. For patients with diabetic nephropathy, the eligible eGFR should be either between $15 \mathrm{~mL} / \mathrm{min} / 1.73 \mathrm{~m}^{2}$ and $59 \mathrm{~mL} / \mathrm{min} / 1.73 \mathrm{~m}^{2}$ or $\geq 60$ $\mathrm{mL} / \mathrm{min} / 1.73 \mathrm{~m}^{2}$ with 24 -h urinary protein $\geq 3.5 \mathrm{~g}$ or urinary albumin-to-creatinine ratio (ACR) $\geq 2,000 \mathrm{mg} / \mathrm{g}$ or equivalent levels of other proteinuria measurements. For patients with neither glomerulonephritis nor diabetic nephropathy, eGFR between $15 \mathrm{~mL} /$ $\mathrm{min} / 1.73 \mathrm{~m}^{2}$ and $59 \mathrm{~mL} / \mathrm{min} / 1.73 \mathrm{~m}^{2}$ was set for enrollment. The etiology of CKD was ascertained by physicians based on clinical assessments and/or findings of biopsy. The eligibility of eGFR and proteinuria was estimated based on locally measured values. The disorders should be ascertained by at least 2 occasions separated by $\geq 3$ months. The exclusion criteria included those with CKD caused by systemic inflammatory illness or autoimmune disease, isolated hematuria, hereditary kidney disease, kidney, or other transplantation, treatment with immunosuppressive agents in the preceding 6 months to treat kidney or immune disease, HIV infection and/or diagnosis of AIDS, chronic heart failure with New York Heart Association class III or IV, known diagnosis of cirrhosis, pregnancy or breastfeeding, malignancy treated with chemotherapy within last 2 years, and current participation in clinical trial. In the current analysis, 3,700 participants with complete record of serum creatinine, who finished the baseline examination between November 1, 2011 and December 31, 2016, were included. The C-STRIDE study was conducted in accordance with the Declaration of Helsinki. The study has been approved by the Ethics Committee of Peking University First Hospital (2011 [363]). All participants provided written informed consent.

\section{Outcome Variables}

In C-STRIDE, the prespecified outcomes are new occurrence of ESKD, CV events, and all-cause mortality $[12,13]$. ESKD is de- 
fined as the initiation of maintenance hemodialysis, maintenance peritoneal dialysis, or kidney transplantation. CV events include nonfatal acute myocardial infarction, unstable angina, hospitalization for congestive heart failure, arrhythmia (including resuscitated cardiac arrest, ventricular fibrillation, sustained ventricular tachycardia, paroxysmal ventricular tachycardia, an initial episode of atrial fibrillation or flutter, severe bradycardia, or heart block), cerebrovascular events (including intraparenchymal hemorrhage, subarachnoid hemorrhage, and cerebral infarction), and peripheral vascular diseases. In the current analyses, we also combined $\mathrm{CV}$ events and deaths due to CV diseases (CVDs) (the International Classification of Disease codes I00-I99) together as composite CV events. Outcomes were investigated at a 3-6 month interval through phone calls made by research assistant staff to the patients or their proxies or through routine outpatient clinical follow-up. The patients who cannot be got in touch with for $>6$ months were labeled as loss of follow-up, with the date of the last follow-up used for censoring. Once the suspected outcomes were recorded, medical records or death certificates were requested to verify the outcomes. An independent committee consisting of specialist physicians in the coordinating center in Peking University First Hospital adjudicated the outcomes. If several CV events or different modes of kidney replacement therapy (KRT) occurred, the first event was used as the index event. Because we focused on the disease prognosis only among patients with CKD stage 1-4, an occurrence of ESKD will lead to a termination of follow-up for the specific patients. The outcomes were followed up through December 31,2017 to guarantee at least 1 year of follow-up for the participants, censoring for all the outcomes.

\section{Measurements}

Body mass index (BMI) was calculated as weight in kilograms divided by the square of height in meters $\left(\mathrm{kg} / \mathrm{m}^{2}\right)$. Overweight was defined as a BMI $\geq 25 \mathrm{~kg} / \mathrm{m}^{2}$. Blood pressure was measured 3 times at 5 -min intervals by a sphygmomanometer at the follow-up visit. The mean value of the 3 readings was calculated. Diabetes was defined as either a fasting blood glucose $\geq 7.0 \mathrm{mmol} / \mathrm{L}$ or a self-reported history of diabetes. A history of CVD covered the diseases of myocardial infarction, hospitalization for congestive heart failure, serious cardiac arrhythmia, peripheral arterial disease, or cerebrovascular events at baseline. Reporting of history of CVD was based on both self-report of patients and review of their medical records by trained staff at the baseline interview. Blood, spot urine, and 24-h urine specimens were collected locally at each participating center and then transported by cold chain to the central laboratory of Peking University First Hospital after simple processing, where specimens were stored at $-80^{\circ} \mathrm{C}$ before being used. Measurements of all serum and urine biomarkers were finished centrally at Peking University First Hospital. The measurements of serum and urine creatinine were traceable to the isotope dilution mass spectrometry. eGFR was determined with the CKD-EPI creatinine equation [14]. The ACR (mg/g creatinine) was calculated. The classification for the eGFR and ACR was determined according to the Kidney Disease Improving Global Outcomes guideline [15].

\section{Statistical Analyses}

Baseline characteristics of the participants were summarized as mean \pm SD for continuous variables in normal distribution and median (interquartile range) for those in skewed distribution, as- certained by the absolute value of either skewness or kurtosis $\geq 3$. Frequency (percentage) was provided for categorical variables. Comparison for the distribution of continuous variables was conducted by $t$ tests or Mann-Whitney $U$ tests, as appropriate, and by $\chi^{2}$ tests for categorical variables. Crude incidence rates were computed for the outcomes as the ratio of the number of events to the total patient-years of follow-up and expressed as the number of events per 100 patient-years. Follow-up time for the events started from baseline and ended at the occurrence of either of the 3 studied outcomes, or otherwise censored at death (for ESKD and CV events), lost of follow-up or on December 31, 2017. The comparison of incidence rates was performed by using the log-rank test. The ratios of crude incidence were computed by dividing the crude incidence rate of ESKD by that of CV events or that of death. The bootstrap method (with 1,000 independent bootstrap samples) was applied to estimate the $95 \%$ confidence intervals (CIs) of the ratios of crude incidence in the total population. Separate bootstrap analyses (each with 1,000 independent bootstrap samples) were conducted among the subsets stratified by a set of well-established risk factors for $\mathrm{CKD}$, including age, sex, history of CVD, eGFR, ACR, and causes of CKD.

The subdistribution Cox proportional hazards regression model was used to determine the association of the risk factors with each of the outcomes, considering competing risk between the outcomes [16]. Death was designated as a competing risk event for ESKD, while both death and ESKD were treated as competing risk events for $\mathrm{CV}$ events. Collinearity between the independent variables was tested by calculating variance inflation factors. Before being entered into the regression model, missing data were imputed with multiple imputation technique with 25 imputations. The regression method was used to impute missing data for continuous variables with normal distribution, the predictive mean matching method for variables with skewed distribution, while logistic regression for categorical variables with binary or ordinal responses. Each imputed dataset was analyzed separately and then results were pooled together. The total percentage of individuals imputed was $49.3 \%(1,825 / 3,700)$. Results were expressed as hazard ratios with $95 \%$ CI. We further depicted cumulative incidence curves for the 3 outcomes stratified by the risk factors, where significant heterogeneity was detected and covariates were adjusted as in the multivariable Cox regression model. Gray's test was given for comparison of the cumulative incidence curves [17]. Two-sided $p$ values $<0.05$ were considered statistically significant. The statistical analysis was performed using SAS software (version 9.4; SAS Institute, San Diego, CA, USA) and Stata software (version 14.2; StataCorp LLC, College Station, TX, USA).

\section{Results}

Totally, 3,700 patients with CKD were included in the analysis, with a mean age of $50 \pm 14$ years and male predominance $(58.2 \%)$. The majority of the participants had $\mathrm{eGFR}<60 \mathrm{~mL} / \mathrm{min} / 1.73 \mathrm{~m}^{2}(70.6 \%)$ and presence of albuminuria (ACR $\geq 30 \mathrm{mg} / \mathrm{g}$ ) (86.1\%). The demographics, clinical risk factors, and indicators for CKD among the total population and stratified by eGFR levels are listed in 
Table 1. Baseline characteristics of the participants for the C-STRIDE

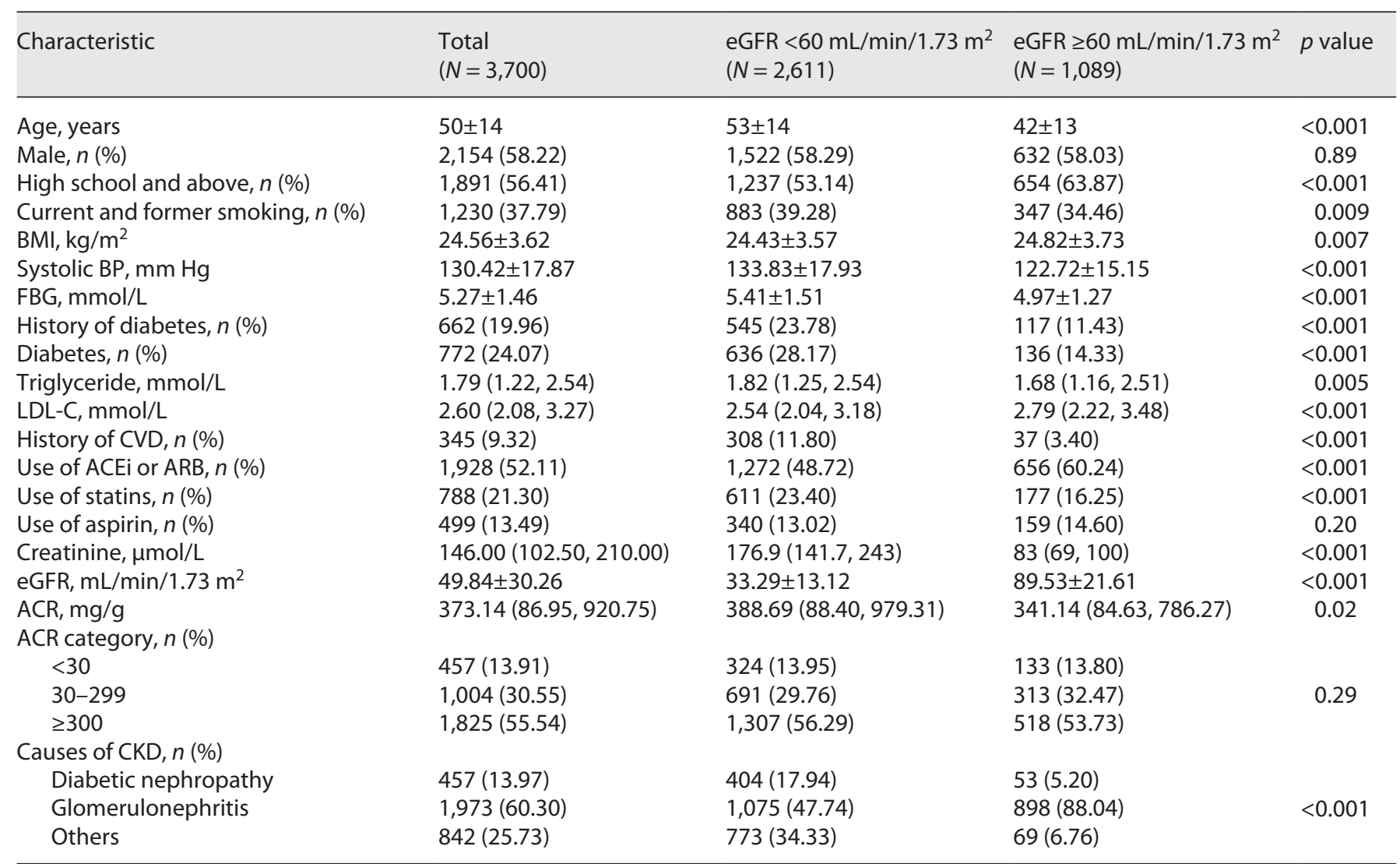

Data were presented as mean \pm standard deviation or median (IQR) for continuous variables according to their distribution and frequency (percentage) for categorical variables. Missing counts: education-348, smoking-445, systolic BP-558, BMI-807, FBG-571, history of diabetes-384, diabetes-493, triglyceride-742, LDL-C-855, ACR-414, and causes of CKD-428. BMI, body mass index; BP, blood pressure; FBG, fasting blood glucose; LDL-C, low density lipoprotein cholesterol; CVD, cardiovascular disease; ACEi, angiotensin-converting enzyme inhibitor; $A C R$, angiotensin receptor blocker; eGFR, estimated glomerular filtration rate; $A C R$, albumin-to-creatinine ratio; CKD, chronic kidney disease; IQR, interquartile range.

Table 1. Patients with reduced kidney function were older; had a lower level of BMI; a higher level of systolic blood pressure, fasting blood glucose, triglyceride, low-density lipoprotein cholesterol, and ACR; more likely to be smokers; have a history of diabetes or CVD, be under treatment of statins; and be attributed to diabetic nephropathy and other causes of CKD but less likely to be under treatment of angiotensin-converting enzyme inhibitor or angiotensin receptor blocker (all $p$ values $<0.05$ ). Patients with missing data for any variable $(n=1,825)$ were slightly older ( $51 \pm 15$ vs. $49 \pm 14$ years), with more female ( $43.95 \%$ vs. $39.68 \%)$ and with lower eGFR (48.04 \pm 30.99 vs. 51.59 $\pm 29.45 \mathrm{~mL} / \mathrm{min} / 1.73 \mathrm{~m}^{2}$ ) than those with complete data (all $p$ values $<0.05$ ).

In total, 203 (5.49\%) patients were lost of follow-up. The median follow-up time for ESKD, nonfatal CV events, and death were 4.65 years (interquartile range: $3.71-5.60$ years), 4.76 years (3.97-5.76 years), and 4.84 years (3.97-5.76 years), respectively. The 513 ESKD consisted of 351 hemodialysis, 141 peritoneal dialysis, and 21 kidney transplantations. The 249 nonfatal CV events were derived from 27 cases of myocardial infarction, 39 cases of unstable angina, 103 hospitalizations for congestive heart failure, 14 cases of arrhythmia, 83 cerebrovascular events, and 4 cases of peripheral vascular diseases. There were 163 cases of death, including 70 cases due to CVD. Overall, the crude incidence rate of ESKD was about 2 times that of nonfatal CV events or composite CV events, while $>3$ times that of death. The overall incidence rates and those stratified by the prespecified risk factors are listed in Table 2. 


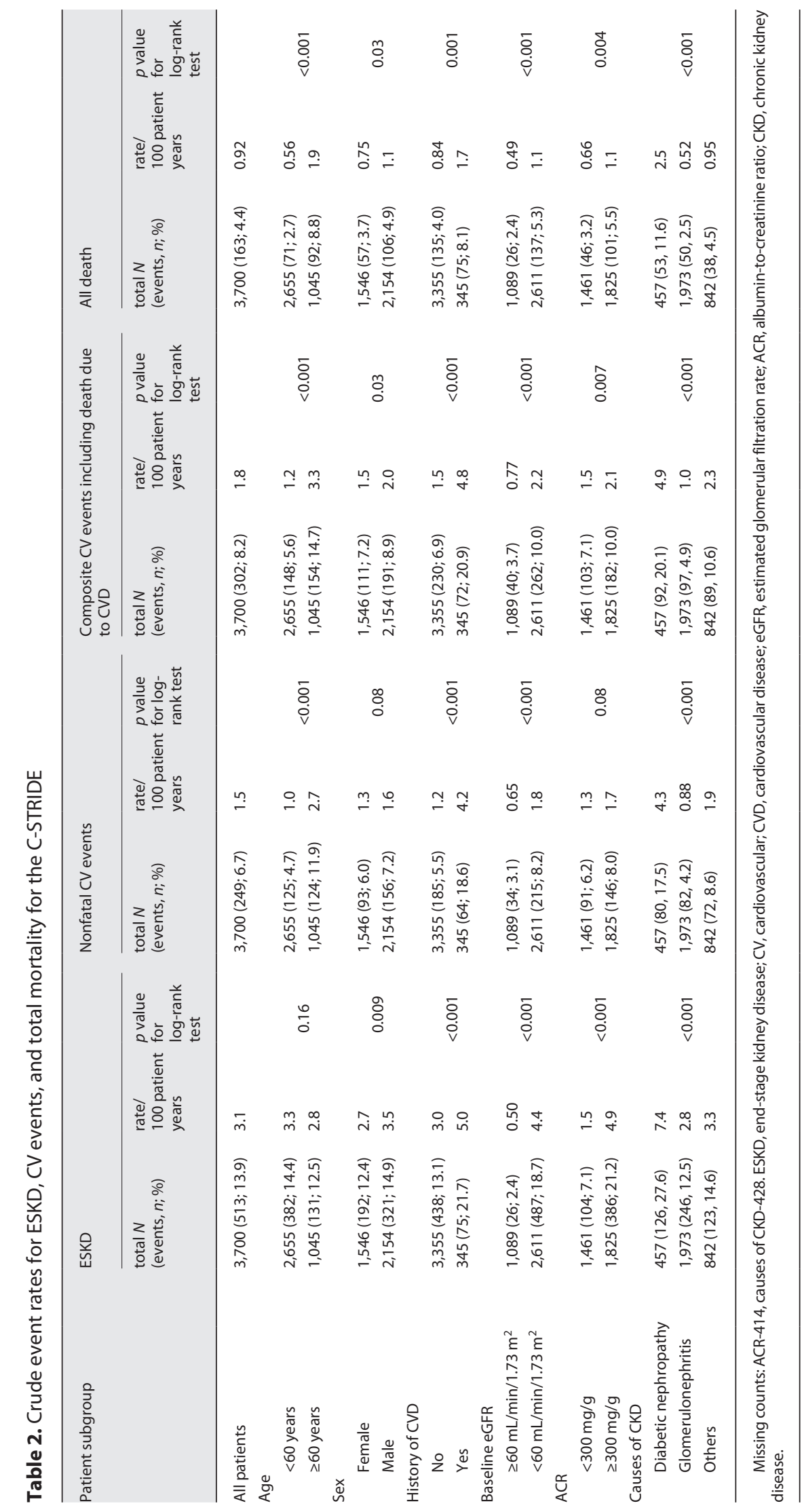


Table 3. Ratios of crude incidence of ESKD, CV events, and total mortality for the C-STRIDE

\begin{tabular}{|c|c|c|c|}
\hline Patient subgroup & $\begin{array}{l}\text { ESKD/nonfatal } \\
\text { CV events* }\end{array}$ & $\begin{array}{l}\text { ESKD/composite } \\
\text { CV events* }\end{array}$ & ESKD/all death* \\
\hline All patients & $2.15(1.87,2.53)$ & $1.77(1.55,2.05)$ & $3.41(2.88,4.08)$ \\
\hline \multicolumn{4}{|l|}{ Age } \\
\hline$<60$ years & $3.25(2.71,3.97)$ & $2.75(2.30,3.33)$ & $5.87(4.69,7.67)$ \\
\hline$\geq 60$ years & $1.07(0.85,1.35)$ & $0.86(0.70,1.06)$ & $1.53(1.17,1.98)$ \\
\hline \multicolumn{4}{|l|}{ Sex } \\
\hline Female & $2.13(1.71,2.75)$ & $1.79(1.46,2.26)$ & $3.61(2.74,4.97)$ \\
\hline Male & $2.17(1.84,2.59)$ & $1.77(1.51,2.10)$ & $3.30(2.71,4.15)$ \\
\hline \multicolumn{4}{|l|}{ History of CVD } \\
\hline No & $2.49(2.11,2.92)$ & $2.01(1.72,2.33)$ & $3.52(2.97,4.24)$ \\
\hline Yes & $1.18(0.86,1.64)$ & $1.05(0.77,1.43)$ & $3.00(2.01,4.72)$ \\
\hline \multicolumn{4}{|l|}{ Baseline eGFR } \\
\hline$\geq 60 \mathrm{~mL} / \mathrm{min} / 1.73 \mathrm{~m}^{2}$ & $0.77(0.43,1.29)$ & $0.65(0.37,1.04)$ & $1.02(0.60,1.71)$ \\
\hline$<60 \mathrm{~mL} / \mathrm{min} / 1.73 \mathrm{~m}^{2}$ & $2.43(2.10,2.86)$ & $1.99(1.74,2.30)$ & $3.99(3.32,4.80)$ \\
\hline \multicolumn{4}{|l|}{$A C R$} \\
\hline$<300 \mathrm{mg} / \mathrm{g}$ & $1.15(0.88,1.53)$ & $1.01(0.78,1.34)$ & $2.33(1.70,3.31)$ \\
\hline$\geq 300 \mathrm{mg} / \mathrm{g}$ & $2.89(2.45,3.53)$ & $2.31(1.98,2.77)$ & $4.36(3.58,5.41)$ \\
\hline \multicolumn{4}{|l|}{ Causes of CKD } \\
\hline Diabetic nephropathy & $1.73(1.32,2.28)$ & $1.51(1.16,1.98)$ & $2.90(2.15,4.03)$ \\
\hline Glomerulonephritis & $3.16(2.52,4.10)$ & $2.67(2.17,3.42)$ & $5.35(4.11,7.40)$ \\
\hline Others & $1.76(1.35,2.34)$ & $1.43(1.11,1.86)$ & $3.47(2.47,5.29)$ \\
\hline
\end{tabular}

Missing counts: ACR-414, causes of CKD-428. ESKD, end-stage kidney disease; CV, cardiovascular; CVD, cardiovascular disease; eGFR, estimated glomerular filtration rate; ACR, albumin-to-creatinine ratio; CKD, chronic kidney disease; $\mathrm{Cl}$, confidence interval. * Results were presented as ratios of crude incidence and $95 \% \mathrm{Cl}$. The total number of cases for ESKD, nonfatal CV events, composite CV events, and death is 513,249, 302, and 163, respectively.

The ratios of crude incidence for the ESKD/nonfatal $\mathrm{CV}$ events (or the ESKD/composite CV events) were significantly different across age-groups, history of CVD, categories of eGFR, categories of ACR, and etiologic causes of CKD (diabetic nephropathy vs. glomerulonephritis), with 95\% CI of the ratios of crude incidence not overlapping among the categories of the variables. The ratios of crude incidence of ESKD/death were significantly different across age-groups, categories of eGFR, categories of ACR, and etiologic causes of CKD (Table 3).

Table 4 listed the results of the association analysis for each of the outcomes. In multivariable-adjusted model, age was significantly associated with increased risk of $\mathrm{CV}$ events and death but associated with reduced risk of ESKD. Reduced eGFR and increased ACR had stronger effects on ESKD than did on other events. The variance inflation factors were all $<4$ in the multivariable analysis, indicating low influence of collinearity. Multivariable adjusted cumulative incidence curves were plotted stratified by age, history of CVD, eGFR, ACR, and causes of CKD. Patients with higher age ( $\geq 60$ years) were associ- ated with a lower cumulative incidence of ESKD but higher cumulative incidence of composite CV events and death than those with lower age ( $<60$ years) (all $p$ values $<0.05$ ) (shown in online suppl. Fig. 1; see www. karger.com/doi/10.1159/000519190 for all online suppl. material). A history of CVD was associated with a higher risk of ESKD, nonfatal, and composite CV events (all $p$ values $<0.05$ ), rather than death (shown in online suppl. Fig. 2). An eGFR $<60 \mathrm{~mL} / \mathrm{min} / 1.73 \mathrm{~m}^{2}$ was associated with a higher cumulative incidence of ESKD ( $p$ value $<0.05$ ), rather than $\mathrm{CV}$ events or death (shown in online suppl. Fig. 3). Similarly, an ACR $>300 \mathrm{mg} / \mathrm{g}$ was significantly associated with a higher cumulative incidence of ESKD and death (both $p$ values $<0.05$ ), while no association was detected for nonfatal or composite CV events (shown in online supp. Fig. 4). Compared with glomerulonephritis, diabetic nephropathy was associated with a significantly higher cumulative incidence of ESKD, $\mathrm{CV}$ events, and death (all $p$ values $<0.05$ ) (shown in online suppl. Fig. 5). 


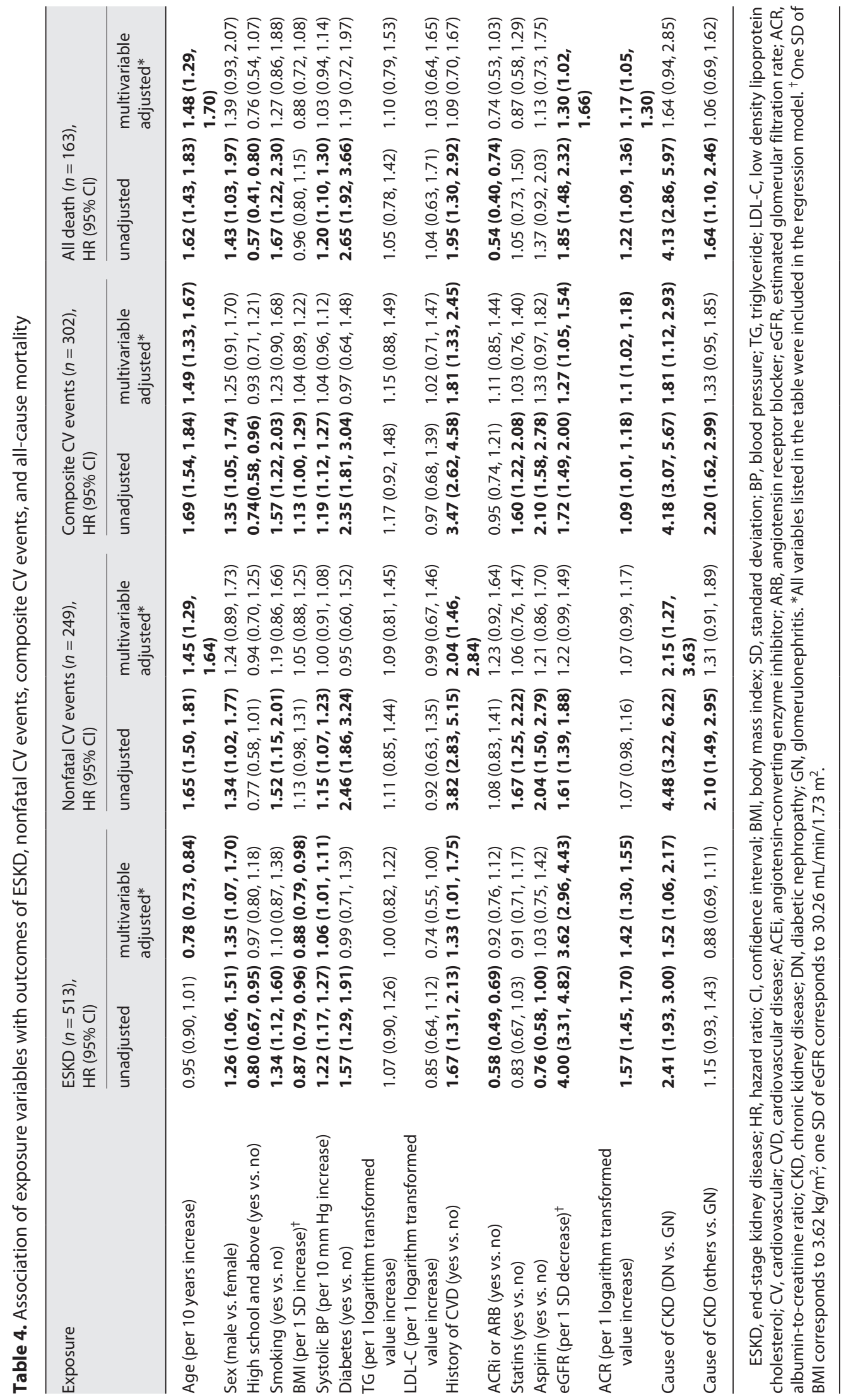




\section{Discussion}

Participants with CKD in the C-STRIDE study were more likely to progress to ESKD than to have CV complications or to die during a median follow-up of nearly 5 years. The comparative risk for different outcomes shows heterogeneity across levels of age, history of CVD, eGFR, albuminuria, and causes of CKD probably because of the different impact of the risk factors on the outcomes.

Based on a 5\% sample of the US Medicare participants during 1998-1999, Foley and colleagues [18] reported a substantially higher rate of congestive heart failure, atherosclerotic vascular disease, and death than KRT after 2 years of follow-up among those with CKD. Similar results were reported by Keith and colleagues [2], based on data of a managed health-care plan with a considerably large sample size $(n=27,998)$. After 5 years of follow-up, the proportion of incident death was remarkably higher than incident ESKD through severely reduced eGFR $\left(15-29 \mathrm{~mL} / \mathrm{min} / 1.73 \mathrm{~m}^{2}\right)$ to normal eGFR $(60-89 \mathrm{~mL} /$ $\mathrm{min} / 1.73 \mathrm{~m}^{2}$ ). However, the participants in their studies were older (exclusively $\geq 65$ years in Foley's study and mean age of 61.4-73.6 years in Keith's study) and had a higher prevalence of CV comorbidities $(21.8 \%$ had atherosclerotic vascular disease and $13.8 \%$ had congestive heart failure in Foley's study and $>40 \%$ had one or more CV comorbidity in Keith's study) than ours (the mean age was $49.94 \pm 14.30$ years and the proportion of history of CVD was $9.32 \%$ ), which may lead to a greater susceptibility for CV events and death in their studied populations $[2,18]$.

In contrast, comparatively higher risk of ESKD than $\mathrm{CV}$ events and death has been reported by several cohort studies recruiting patients with CKD. In the AASK study, the incidence rate of ESKD exceeded that of all-cause mortality, CVD mortality, and composite CVD events among African Americans with hypertensive nephrosclerosis after a total follow-up of 11 years. However, the AASK study was initially a clinical trial, which recruited patients of hypertensive CKD with disproportionately low risk of CVD such as a low prevalence of DM (5.3\%) and exclusion of those with previous CVD [6]. In the Chronic Renal Insufficiency Cohort study, which recruited patients from several clinics and with different ethnicities in the USA, incidence rates of ESKD or halving of eGFR also exceeded composite CV events and death [19]. Among Asians, the multicenter CKD-Japan cohort study enrolling patients with CKD in Japan found that more patients initiated KRT before having a CV event or death [7]. As recruited from clinics with well management for metabolic risk factors, these patients may be more likely to reach ESKD than having onset of CV events or death.

Diabetic nephropathy is the main type of etiology of CKD in developed countries, which typically contributes to $>50 \%$ of ESKD among patients with CKD [20]. However, patients with diabetic nephropathy were often older and have more comorbidities than those with glomerulonephritis. In our study, although diabetic nephropathy was associated with an increased risk of both ESKD and $\mathrm{CV}$ events compared with glomerulonephritis, ESKD was comparatively less likely to happen than $\mathrm{CV}$ events, reflected by lower ratios of crude incidence of ESKD/CV events, among patients with diabetic nephropathy than those with glomerulonephritis. As the majority of patients in our study have the etiology of glomerulonephritis, it is reasonable to expect that more patients will reach ESKD than do patients with the majority of diabetic nephropathy. In addition, patients with glomerulonephritis are typically younger than those affected by diabetic nephropathy or hypertensive nephrosclerosis. This may partly explain the findings of our study that increased age is protective for risk of ESKD. A recent study has reported that advanced age, even in the absence of metabolic comorbidities, will lead to a significantly reduced level of measured GFR [21]. With the aging of the population and the improved accessibility to the dialysis service, the incidence of ESKD is likely to increase in China. However, other risk factors, especially the metabolic disorders, may also be taken into account to explain the comparative risk between the outcomes. For example, as mentioned above, much higher rates of $\mathrm{CV}$ events and death were observed than ESKD in Foley's study, which recruited older adults and those with glomerulonephritis as the dominant etiology of CKD (58.7-60.2\%) [18].

Several countries/regions in north and east Asia have the highest burden of ESKD around the world, with the prevalence of ESKD of 3,219.4, 2,504.8, and 1,571.5 per million population in Taiwan, Japan, and South Korea, respectively [22]. Comparatively, the prevalence of ESKD was much lower in mainland China. A research based on the nationwide health insurance database showed the prevalence of ESKD in the country is only 402.18 per million population in 2015 [8]. Based on the local registry for dialysis, a study in Shanghai, which has very good reimbursement policy for dialysis, showed that the prevalence of hemodialysis and peritoneal dialysis was 898.2 per million population in 2014 [9]. The prevalence of diabetes is increasing in China since 1980s, probably leading to the increasing burden of diabetic nephropathy [23]. Actually, 
a recent published study based on the national hospitalization database reported that proportion of CKD related to diabetes surpassed that of CKD related to glomerulonephritis in 2011 and the gap between the 2 etiologies kept enlarging after the year [24]. So, it can be foreseen that $\mathrm{CV}$ events may be more commonly seen among patients with CKD in China in the future.

There are some limitations of the study. First, although enrolled from around China, the participants were all from outpatients in local central clinical centers, who were under well management for the metabolic disorders. Hence, the participants may not represent the general population with $\mathrm{CKD}$, the majority of whom may not be aware of their condition and seek medical care. Second, the median follow-up time for the participants was about 5 years. Given the much longer time needed to reach an endpoint for CKD, the follow-up time may lead to a limited number of endpoints, potentially restricting the statistical power of the study. Third, underreporting of the outcomes cannot be ruled out. However, the extent for the missing might be low, since incidence rates of the outcomes were largely comparable between the present study and other cohorts recruiting similar participants.

In conclusion, our study found the higher incidence rate of ESKD than CV events or death in a multicenter prospective cohort study with a large number of patients with stage 1-4 CKD and a relatively long period of followup. Age, history of CVD, eGFR, urine ACR, and etiology of $\mathrm{CKD}$ were shown to exert a heterogeneous influence on the risk of the outcomes. Further studies are warranted to verify our findings and evaluate the potentially beneficial effect of modifying the risk factors on the outcomes of CKD.

\section{Acknowledgments}

The authors would like to express gratitude to every participating investigator of the C-STRIDE group for their collaboration.

\section{Statement of Ethics}

All participants have given written informed consent. The study has been approved by the Ethics Committee of Peking University First Hospital (2011[363]).

\section{Conflict of Interest Statement}

Prof. Ming-Hui Zhao is an editorial board member of Kidney Diseases. The other authors have no conflicts of interest to declare.

\section{Funding Sources}

This study was supported by grants from the National Natural Science Foundation of China (91846101, 81771938, 81301296, and 81900665), Beijing Nova Programme Interdisciplinary Cooperation Project (Z191100001119008), the National Key R\&D Program of the Ministry of Science and Technology of China (2016YFC1305405, 2019YFC2005000), Chinese Academy of Medical Sciences Research Unit (No. 2019RU023), CAMS Innovation Fund for Medical Sciences (2019-I2M-5-046), Chinese Scientific and Technical Innovation Project 2030 (2018AAA0102100), the University of Michigan Health System-Peking University Health Science Center Joint Institute for Translational and Clinical Research (BMU20160466, BMU2018JI012, and BMU2019JI005), PKU-Baidu Fund (2019BD017), and from Peking University (BMU2018MX020, PKU2017LCX05). The funders had no role in the study design; collection, analysis, and interpretation of data; writing the report; and the decision to submit the report for publication.

\section{Author Contributions}

J.W., J.L., K.H., and L.Z. designed the study; J.W., F.W., B.G., M.-H.Z., and L.Z. collected the data; J.W. and J.L. analyzed the data; J.W., K.H., F.W., B.G., M.-H.Z., and L.Z. drafted and revised the paper; all authors approved the final version of the manuscript.

\section{Data Availability Statement}

The datasets generated and analyzed during the current study are available from the corresponding author on reasonable request.

\section{References}

1 Jha V, Garcia-Garcia G, Iseki K, Li Z, Naicker S, Plattner B, et al. Chronic kidney disease: global dimension and perspectives. Lancet. 2013;382(9888):260-72.

2 Keith DS, Nichols GA, Gullion CM, Brown JB, Smith DH. Longitudinal follow-up and outcomes among a population with chronic kidney disease in a large managed care organization. Arch Intern Med. 2004;164(6):65963.
3 Sarnak MJ, Levey AS, Schoolwerth AC, Coresh J, Culleton B, Hamm LL, et al. Kidney disease as a risk factor for development of cardiovascular disease: a statement from the American Heart Association councils on kidney in cardiovascular disease, high blood pressure research, clinical cardiology, and epidemiology and prevention. Circulation. 2003;108(5):2154-69.
4 Foley RN, Parfrey PS, Sarnak MJ. Clinical epidemiology of cardiovascular disease in chronic renal disease. Am J Kidney Dis. 1998; 32(5 Suppl 3):S112-9.

5 Orlandi PF, Huang J, Fukagawa M, Hoy W, Jha $\mathrm{V}$, Oh $\mathrm{KH}$, et al. A collaborative, individual-level analysis compared longitudinal outcomes across the International Network of Chronic Kidney Disease (iNETCKD) cohorts. Kidney Int. 2019;96(5):1217-33. 
6 Alves TP, Wang X, Wright JT Jr, Appel LJ, Greene T, Norris K, et al. Rate of ESRD exceeds mortality among African Americans with hypertensive nephrosclerosis. J Am Soc Nephrol. 2010;21(8):1361-9.

7 Tanaka K, Watanabe T, Takeuchi A, Ohashi Y, Nitta K, Akizawa T, et al. Cardiovascular events and death in Japanese patients with chronic kidney disease. Kidney Int. 2017;91(1):227-34.

8 Wang F, Yang C, Long J, Zhao X, Tang W, Zhang D, et al. Executive summary for the 2015 annual data report of the china kidney disease network (CK-NET). Kidney Int. 2019; 95(3):501-5.

9 Chen Z, Zhang W, Chen X, Hsu CY. Trends in end-stage kidney disease in Shanghai, China. Kidney Int. 2019;95(1):232.

10 Gao B, Zhang L, Wang H, Zhao M. Chinese cohort study of chronic kidney disease: design and methods. Chin Med J. 2014;127(11):2180-5.

11 Wang Q, Wang Y, Wang J, Zhang L, Zhao MH; C-STRIDE (Chinese Cohort Study of Chronic Kidney Disease). Short-term systolic blood pressure variability and kidney disease progression in patients with chronic kidney disease: results from C-STRIDE. J Am Heart Assoc. 2020;9(12):e015359.

12 Lv L, Wang J, Gao B, Wu L, Wang F, Cui Z, et al. Serum uromodulin and progression of kid- ney disease in patients with chronic kidney disease. J Transl Med. 2018;16(1):316.

13 Yuan Q, Wang J, Peng Z, Zhou Q, Xiao X, Xie $\mathrm{Y}$, et al. Neutrophil-to-lymphocyte ratio and incident end-stage renal disease in Chinese patients with chronic kidney disease: results from the Chinese cohort study of chronic kidney disease (C-STRIDE). J Transl Med. 2019; 17(1):86.

14 Levey AS, Stevens LA, Schmid CH, Zhang YL, Castro AF 3rd, Feldman HI, et al. A new equation to estimate glomerular filtration rate. Ann Intern Med. 2009;150(9):604-12.

15 Stevens PE, Levin A. Evaluation and management of chronic kidney disease: synopsis of the kidney disease: improving global outcomes 2012 clinical practice guideline. Ann Intern Med. 2013;158(11):825-30.

16 Li L, Yang W, Astor BC, Greene T. Competing risk modeling: time to put it in our standard analytical toolbox. J Am Soc Nephrol. 2019;30(12):2284-6.

17 Austin PC, Fine JP. Practical recommendations for reporting Fine-Gray model analyses for competing risk data. Stat Med. 2017; 36(27):4391-400.

18 Foley RN, Murray AM, Li S, Herzog CA, McBean AM, Eggers PW, et al. Chronic kidney disease and the risk for cardiovascular disease, renal replacement, and death in the United States medicare population, 1998 to 1999 . J Am Soc Nephrol. 2005;16(2):489.

19 Denker M, Boyle S, Anderson AH, Appel LJ, Chen J, Fink JC, et al. Chronic renal insufficiency cohort study (CRIC): overview and summary of selected findings. Clin J Am Soc Nephrol. 2015;10(11):2073-83.

20 Thomas MC, Cooper ME, Zimmet P. Changing epidemiology of type 2 diabetes mellitus and associated chronic kidney disease. Nat Rev Nephrol. 2016;12(2):73-81.

21 Eriksen BO, Palsson R, Ebert N, Melsom T, van der Giet M, Gudnason V, et al. GFR in healthy aging: an individual participant data meta-analysis of iohexol clearance in European population-based cohorts. J Am Soc Nephrol. 2020;31(7):1602-15.

22 Wang J, Zhang L, Tang SC, Kashihara N, Kim YS, Togtokh A, et al. Disease burden and challenges of chronic kidney disease in North and East Asia. Kidney Int. 2018;94(1):22-5.

23 Wang L, Gao P, Zhang M, Huang Z, Zhang D, Deng Q, et al. Prevalence and ethnic pattern of diabetes and prediabetes in China in 2013. JAMA. 2017;317(24):2515-23.

24 Zhang L, Long J, Jiang W, Shi Y, He X, Zhou $Z$, et al. Trends in chronic kidney disease in China. N Engl J Med. 2016;375(9):905-6. 